\title{
Virulence traits contributing to pathogenicity of candida species
}

\begin{abstract}
Candida is the unique opportunistic mycotic pathogen that has adapted variety of mechanisms to establish itself both as commensal and pathogen in humans. This yeast like fungus presents in many clinical forms, ranging from superficial manifestations involving the skin, nails and mucosal surfaces to deep seated infections involving various internal organs and disseminated diseases. Although Candida spp. can initiate infection in both immunocompetent and immunocompromised hosts, the incidence of candidiasis is usually high in immunocompromised patients. Therefore the role of Candida in overall process of initiation and progression of infection was considered to be passive. However, recently this concept is revamped and it can be now stated that Candida actively participates in the pathophysiology of establishment and progression of infection through the mechanisms of aggression known as virulence factors. Some of these virulence factors help in colonization or initiation of infection while others aid in progression of infection or dissemination in host tissues. Adherence to host tissues, biofilm formation on medical devices and secretion of ectohydrolases are some of putative virulence traits of Candida spp. Although virulence factors contributing to pathogenicity of Candida albicans is well studied, the search through available literature has revealed a dearth of information on virulence factors of non albicans Candida (NAC) spp. The identification of virulence attributes unique to a particular Candida spp. is very important to understand the pathogenesis and epidemiology of candidiasis.
\end{abstract}

Keywords: adhesion, biofilms, extracellular hydrolytic enzymes, morphogenesis, phenotypic switching, virulence traits
Volume 5 Issue I - 2017

\author{
Sachin C Deorukhkar, Shahriar Roushani \\ Department of Microbiology, Rural Medical College, Pravara \\ Institute Medical Sciences, India
}

\begin{abstract}
Correspondence: Sachin C Deorukhkar, Department of Microbiology, Rural Medical College, Pravara Institute Medical Sciences (Deemed University), Loni, Maharashtra, India, Tel 919545।81908; +91-9850775564, Fax 91-2422-273442, Email deorukhkar.sachin@gmail.com
\end{abstract}

Received: February 27, 2017| Published: June 14, 2017

\section{Introduction}

The fungus belonging to genus Candida is the only opportunistic mycotic pathogen causing a wide spectrum of clinical manifestations ranging from mucocutaneous overgrowth to life threatening disseminated infections. ${ }^{1}$ This opportunistic fungal pathogen is ubiquitous in nature and can exist as saprophyte and commensal. However, under certain conditions Candida may transit from a commensal to a potent pathogen.

The transition of Candida from a harmless commensal to potent pathogen is contributed by various host factors and pathogenicity of infecting Candida spp. As candidiasis is frequently encountered in immunocompromised and critically ill immunocompetent individuals the role of Candida in overall process of initiation and progression of infection was considered to be passive. Therefore, organic weakness or a compromised immune status of the host was considered as the only mechanism responsible for establishment of this opportunistic mycotic infection. ${ }^{2}$ However, recently this concept is revamped and it can be now stated that Candida actively participate in the pathophysiology of establishment and progression of infection through the mechanisms of aggression known as virulence factors. ${ }^{2,3}$ Virulence factors can be defined as all traits produced by infecting strain of pathogen to establish the process of infection in the host.

The pathogenicity of Candida spp. can be attributed to various virulence traits like adhesion to host tissues and surface of medical devices, biofilm formation and secretion of extracellular hydrolases, phenotypic switching and thigmotropism. ${ }^{3,4}$ These virulence traits of infecting Candida spp. directly interact with host cells and leads to tissue damage. ${ }^{5}$ Although virulence factors contributing to pathogenicity of Candida albicans is well studied, the search through available literature has revealed a dearth of information on virulence factors of non albicans Candida (NAC) spp. once ignored as commensal or disseminated as a mere contaminant in clinical specimens, have emerged as an important cause of infections. ${ }^{6}$ Many researchers from different parts of the world have reported predominance of NAC spp. over C. albicans in different clinical types of Candida infections. In this research, various virulence factors contributing to the pathogenicity of Candida spp. with emphasis on 'cryptic' NAC spp. was investigated and concisely presented in this review.

\section{Methodology}

For preparation of this review article, relevant original and review articles were retrieved from search engines like 'PubMed' and 'Google Scholar'. The search was made using MeSH terms like 'virulence factors of Candida', 'pathogenicity of Candida species' and 'pathogenic features of Candida'.

\section{Discussion}

In Candida spp. combination of different virulence factors contributes at each stage of colonization and infection. At some stages a particular virulence factor may be dominant over the other.

\section{Adhesion}

The versatility and ability of Candida spp. to adhere and survive on a variety of human tissues makes it an important organism among fungal pathogens. Adhesion to the tissue is the primary and most important stage in the process of Candida colonization and infection. ${ }^{7}$ Candida spp. can adhere to various host cells like epithelia, endothelia and phagocytes. ${ }^{8}$ Adherence to host cells confers certain 
important properties to infecting Candida spp. Adhesion prevents or at least decreases the extent of clearance by defense mechanism of the host. It also ensures the delivery of Candidial enzymes and toxins to the host's cell targeted. ${ }^{3}$. Adhesion helps Candida cells to penetrate, disseminate and persist in host tissues. ${ }^{3}$

Similar to other microbial pathogens, adhesion in Candida spp. is initiated and controlled by several cell-signaling cascades. The process of adherence to host cell is initiated by non-specific factors like hydrophobicity and electrostatic forces and further preceded by specific adhesins present on the surface of Candida cells. ${ }^{9}$ Adhesins are surface proteins involved in specific adherence. ALS (agglutininlike sequence) family, Hwp (hyphal wall protein) 1, Int (Integrin like surface protein) 1 , Mnt ( $\alpha$-1-2-mannosyltransferase) 1 and EPA (epithelial adhesin) 1 are examples of well characterized adhesins of Candida spp. ${ }^{8,10}$. Candidial adhesins recognizes and binds to ligands like proteins, fibrinogen and fibronectin. As adhesins like Als3 and Hwp1 are predominately expressed during the process of hypha formation, they play important role in the adhesion of $C$. albicans to host cells. ${ }^{8}$ In C. glabrata (the only known haploid Candida spp. that lack ability to form hyphae or pseudohyphae) EPA gene family encodes for major group of adhesins. Epa proteins are structurally similar to that of Als proteins of $C$. albicans. ${ }^{11}$. In case of $C$. parapsilosis, genes for five Als proteins and six for pga 30 (predicted glycosylphosphotidylinositol-anchored protein 30) have been identified. ${ }^{11}$ At least three Als proteins have been identified in cell wall of $C$. tropicalis through western blot analysis. ${ }^{11}$

The process of adhesion is influenced by various factors like the type of cell wall proteins and physicochemical properties of cell surface. Three types of interactions have been proposed for adhesions of Candida spp. to the host cells. ${ }^{12}$. These include proteinprotein interaction, lectin-like interaction and incompletely defined interactions. ${ }^{12}$ Numerous assays like visual cell binding, radiometric binding, whole cell-ligand binding and Candidal adhesion-ligand binding are available for in vitro measurement of Candida adherence.

\section{Biofilm formation}

In recent years, Candida spp. is frequently isolated from nosocomial bloodstream infections, pneumonias and urinary tract infections. ${ }^{13}$ Due to their versatility of adapting to a variety of different habitats including medical devices, Candida spp. have emerged as one of the important causes of health- care associated infections. ${ }^{14}$ Candida rank 3rd among leading causes of catheter-associated infections. ${ }^{15}$ Nearly half of all cases of nosocomial infections are associated with indwelling medical devices. ${ }^{16}$ Medical devices are integral to a patientcentric care continuum, from screening to diagnosis to treatment and monitoring. Candida spp. can adhere, colonize and form biofilm on most, if not all, medical devices in current use. ${ }^{17}$ Biofilms are surfaceassociated microbial communities which are firmly fixed within an extracellular matrix. ${ }^{13}$

Biofilm limits the penetration of an antifungal agent through the matrix and protects the yeast cells from host defense mechanism. Candida biofilms are resistant to inhibitory action exerted by neutrophils. ${ }^{18}$ The mortality rate of infections caused by biofilm forming Candida isolates is significantly higher than that of non biofilm forming strains. The mortality rate of medical device associated Candida infections is as high as $30 \%{ }^{2}$ In Candida spp. the ability to form biofilm and extracellular matrix varies according to species, strain and environmental factors. Biofilm matrix of $C$. albicans composes of carbohydrates, proteins, phosphorus and hexosamines. C. tropicalis biofilm matrix has low levels of carbohydrates and proteins compared to C. albicans and other NAC spp. ${ }^{11}$ C. glabrata biofilm matrix is made of high levels of proteins and carbohydrates. The extracellular matrix of $C$. parapsilosis biofilm is mainly contains carbohydrates and low levels of proteins. ${ }^{11}$ Biofilm formation is noted in NAC spp. like C. tropicalis, C. glabrata, C. parapsilosis and C. dubliniensis. In one of our study, we noted greater biofilm forming ability in C. tropicalis compared to C. albicans. ${ }^{6}$ Various model systems like catheter disc, acrylic disc, microtiter plate, cylindrical cellulose filter, perfused biofilm fermenter and modified Robbins device have been used for characterization and antifungal susceptibilities of Candida biofilms.

\section{Extracellular hydrolytic enzymes}

Extracellular hydrolases play an important role in the pathogenesis of Candida infections. These extracellular hydrolytic enzymes facilitate the invasion of host tissue by deranging constituents of host cell membrane. ${ }^{2}$ Phospholipases, secreted aspartyl proteinases, lipases and hemolysins are the secreted hydrolases most commonly implicated in the pathogenicity of Candida. ${ }^{2}$ In Candida spp., enzyme phospholipase hydrolyses phospholipids of host cell membrane and expose receptors to facilitate the adherence of yeast cells. ${ }^{19}$ Phospholipase activity in C. albicans was first reported by Costa and Werner. ${ }^{3}$ Till date, seven phospholipase genes have been identified in C. albicans. These include PLA, PLB1, PLB2, PLC1, PLC2, PLC3 and PLD $1 .{ }^{19}$

Several researchers have reported phospholipase activity in $C$. albicans and NAC spp. like C. tropicalis and C. parapsilosis. In contrary to C. tropicalis and C. parapsilosis, very few studies are available on phospholipase production in C. glabrata. ${ }^{19} \mathrm{C}$. dubliniensis isolates often demonstrate low phospholipase activity. This could be one of the possible reason for minimal or no role of this NAC spp. in invasive infections. ${ }^{2}$ As phospholipase activity is concentrated at the growing hyphal tip, this enzyme plays a vital role in invasion host tissue especially in disseminated Candida infections.

Several studies have reported association between phospholipase production and other factors of Candida spp. like antifungal resistance and biofilm production. Ying \& Chungyang. ${ }^{20}$ reported co-relation between high phospholipase activity in Candida isolates and resistance to fluconazole..$^{20}$ In one of our study, phospholipase activity was significantly high among biofilm forming Candida isolates. ${ }^{2}$ Therefore screening of phospholipase activity in biofilm forming Candida spp. can serve as an important parameter to differentiate invasive strains from non invasive colonizers. ElFeky et al..$^{21}$ reported significantly high phospholipase activity among Candida spp. isolated from disseminated infections compared to mucocutaneous infections. ${ }^{21}$ Egg yolk agar plate based method is the easy and widely used for screening of phospholipase production in Candida isolates.

Proteinase activity of Candida spp. is due to ten secreted aspartic proteinase (Sap) isoenzymes. ${ }^{22}$ In Candida, Sap is encoded by ten genes SAP1-10. Sap deteriorates epithelial and mucosal barrier proteins such as collagen, keratin and mucin. Additionally, it also degrades complement, cytokines and immunoglobulins. ${ }^{3}$ Sap1-6 is important for adherence, tissue damage and alteration in the host defense mechanisms. ${ }^{3}$ The extracellular proteinase was first described by Staib. ${ }^{3}$ Till date the role of Sap 7 in pathogenicity of Candida spp. is not completely understood whereas, Sap 9 and Sap 10 are important in maintaining the regulatory surface integrity of yeast cells. ${ }^{3}$ Many studies have reported variable proteinase activity among different Candida spp. As compared to other Candida spp., very little is known about proteinase activity of C. glabrata, C. krusei and C. 
kefyr. In one of our study, $C$. dubliniensis isolated from HIV infected patients with oropharyngeal candidiasis showed higher proteinase activity compared to C. albicans. ${ }^{6}$ Till date, three SAP genes (SAPP13) have been identified in C. parapsilosis. ${ }^{11}$ Four SAP genes (SAPT14) have been identified in C. tropicalis. C. tropicalis demonstrates high proteinase activity in medium containing bovine serum albumin as the sole source of nitrogen. ${ }^{11}$

Sometimes proteinase antigen is expressed on fungal elements engulfed by phagocytes. The proteinase production within the phagolysosome degrades proteins involved in the process of generation of candidacidal oxygen radicals. ${ }^{23}$ Various factors like strain type, type of infection, phenotypic switch type, environmental conditions and stage of infection affect the proteinase production. The role of Saps in pathogenesis of disseminated candidiasis appears to be less important compared to mucocutaneous infections. ${ }^{21}$

In vitro proteinase activity of Candida spp. can be elicited in a culture medium containing exogenous protein like bovine serum albumin, hemoglobin, keratin, collagen, or complex peptide mixtures like peptone and tryptone as a sole nitrogen source. Sap can be also be detected by spectrophotometry, ELISA and western blotting technique. In Candida spp., hemolysins degrade hemoglobin and facilitate recovery of the elemental iron from host erythrocytes. ${ }^{3}$ As hemolysins enable survival and persistence of Candida in the host, it is considered as one of the putative virulence traits contributing to pathogenicity.

Genetic mechanism for haemolysin production is not completely elucidated for Candida spp., however Luo et al. ${ }^{24}$ reported hemolysin like protein (HLP) gene to be associated with hemolysin production in Candida spp..${ }^{24}$ The extent of hemolysin production is dependent on both species and strain of Candida spp. Hemolysin activity is reported in both $C$. albicans and NAC spp. like $C$. glabrata, $C$. tropicalis, $C$. parapsilosis and C. dubliniensis. Blood agar plate assay is a simple and cost effective method for the detection of in vitro haemolytic activity of Candida spp.

As compared to other enzymes of Candida spp., very few studies are directed towards coagulase. Rodrigues et al. ${ }^{25}$ first reported coagulase activity in Candida spp. ${ }^{25}$. Enzyme coagulase binds to fibrinogen and activates a cascade of reactions that leads to clotting of plasma. Coagulase production in Candida spp. is slow as compared to Staphylococcus aureus. Coagulase activity is reported in $C$. albicans and NAC like C. tropicalis and C. parapsilosis. In one of our study, C. glabarta demonstrated high coagulase activity compared to other NAC spp. Similar finding was reported by Yigit et al. ${ }^{26}$ Rodrigues et al. ${ }^{25}$ reported high coagulase activity in C. tropicalis isolates. ${ }^{25}$

Extracellular lipases facilitate degradation of lipids for nutrient acquisition and adhesion to host tissues. Lipases also nonspecifically initiate inflammatory processes by enhancing cell mediated immunity. ${ }^{27}$. This group of enzymes also alters host defense by lysing competing microflora. Extracellular lipase production in $C$. albicans was first described by Werner (1965). ${ }^{27}$ C. albicans lipases are encoded by 10 genes (LIP1 to LIP10). ${ }^{27}$ Similar sequences are also identified in C. tropicalis. However till date no studies have been conducted to investigate the role of lipase genes in the virulence of $C$. tropicalis.

\section{Polymorphism}

Among various pathogenic yeasts Candida spp. is the only polymorphic fungus. C. albicans can either grow as budding yeast, as elongated ellipsoid cells with constrictions at the septa (pseudohyphae) or as parallel-walled true hyphae. ${ }^{18}$ The production of hyphae is considered as one of the mechanism of virulence as it facilitate tissue invasion and also resists phagocytosis. Apart from $C$. albicans, the novel species $C$. dubliniensis is also capable of growing isotropically (yeast form) or apically (hyphal or pseudohyphal form). Although $C$. tropicalis demonstrates similar morphological forms like $C$. albicans only few studies have explored its role in virulence. Silva et al ${ }^{28}$ reported that only apical forms of $C$. tropicalis are capable of invading oral epithelium..$^{28}$

The transformation of unicellular yeast to multicellular hyphal forms is known as dimorphism. Both yeast and hyphal forms play an important role in the virulence. Although hyphal forms are more invasive than yeast forms, yeast form is believed to be primarily involved in dissemination. This mechanism may explain the involvement of C. glabrata in disseminated candidiasis. C. glabrata is the only Candida spp. that is haploid and lack the ability to produce both true and pseudohyphae.

Many strains of C. albicans are capable of switching spontaneously, reversibly and at high frequencies between numerous general phenotypes distinguishable by colony morphology. ${ }^{2}$ Phenotypic switching is one of the putative virulence attributes of $C$. albicans. Soll et al. ${ }^{29}$ described phenotypic switching in C. albicans. ${ }^{29}$ Phenotypic switching is defined as the ability of microorganisms of a single strain to switch reversibly and at high frequency among different colony phenotypes. It regulates various phenotypic characteristics involved in pathogenesis such as adherence to host cells, expression of cell surface hydrophobicity and secretion of proteinases. Phenotypic switching in Candida spp. due to the regulation of pathogenic phasespecific genes is observed during response to antifungal therapy and alteration in host immune mechanism. ${ }^{30}$ Mane et al. ${ }^{31}$ noted greater phenotypic switching in Candida isolates from HIV infected individuals compared to those from HIV non infected individuals. ${ }^{31}$ Phenotypic switching could be considered analogous to the 'Phase transition' described for bacteria. It also allows rapid adaptation of Candida spp. to different stressful environments. The molecular mechanism involved in phenotypic switching is yet to be elucidated.

In addition to $C$. albicans, NAC spp. like C. dubliniensis also exhibits high frequencies of phenotypic switching and produces numerous colony morphologies. Csank \& Haynes $^{32}$ reported phenotypic switching in C. glabrata. $^{32}$ C. glabrata demonstrated spontaneous, reversible and high frequency phenotypic switching on CuSo4 -containing indicator agar plates. ${ }^{32}$ Till date, there is no study available on the phenotypic switching of $C$. tropicalis and other $N A C$ spp.

\section{Thigomotropism}

The directional hyphal growth demonstrated by Candida spp on surfaces with specific topologies is known as thigomotropism. ${ }^{18}$ Thigomotropism is one of the important mechanisms contributing to the virulence of Candida spp. This process helps in biofilm formation on various abiotic surfaces like medical devices and dissemination into host tissues. Thigomotropism of $C$. albicans hyphae is regulated by extracellular calcium uptake via the calcium channels. ${ }^{18}$ Further studies are needed to explore the role of this virulence trait in the pathogenicity of NAC spp.

\section{Conclusion}

Virulence factors are all traits required for establishment and progression of infection. The pathogenicity of Candida spp. is 
attributed to the number of virulence factors like adhesion to host tissue, biofilm formation and secretion of ectoenzymes. The different virulence factors contribute at each stage of infection. However at some stages one virulence factor may be dominant over other or may act as an antagonist. Therefore, the identification of virulence attributes unique to a particular Candida spp. is very important to understand the pathogenesis and epidemiology of candidiasis.

\section{Acknowledgements}

None.

\section{Conflicts of interest}

None.

\section{Funding}

None.

\section{References}

1. Deorukhkar SC. Immunity to Candida infection: An overview. MOJ Immunol. 2017;5(1):00144.

2. Sardi JCO, Scorzoni L, Bernardi T, et al. Candida species: current epidemiology, pathogenicity, biofilm formation, natural antifungal products and new therapeutic options. J Med Microbiol. 2013;62(pt 1):10-24.

3. Deorukhkar SC, Saini S. Candidiasis: Past, present, future. Int J Infect Trop Dis. 2015;2:12-24.

4. Sachin CD, Ruchi K, Santosh S. In vitro evaluation of proteinase, phospholipase and haemolysin activities of Candida species isolated from clinical specimens. Int J Med Biomed Res. 2012;1(2):153-137.

5. Haynes KA. Virulence in Candida species. Trends Microbiol. 2001;9(12):591-596.

6. Deorukhkar SC, Saini S, Mathew S. Non-albicans Candida infection: An emerging treat. Interdisciplinary Perspectives on Infectious Diseases. 2014:7.

7. Deorukhkar SC, Saini S, Mathew S. Virulence factors contributing to pathogenicity of Candida tropicalis and its antifungal susceptibility profile. Int J Microbiol. 2014:6.

8. Calderone RA, Fonzi WA. Virulence factors of Candida albicans. Trends Microbiol. 2015;9(7):327-335.

9. Li X, Yan Z, Xu J. Quantitative variation of biofilms among strains in natural populations of Candida albicans. Microbiol. 2003;149(Pt 2):353-362.

10. Cannon RD, Chaffin WL. Oral colonization by Candida albicans. Crit Rev Oral Biol Med. 1999;10(3):359-383.

11. Silva S, Negri M, Henriques M, et al. Candida glabrata, Candida parapsilosis and Candida tropicalis: biology, epidemiology, pathogenicity and antifungal resistance. FEMS Microbiol Rev. 2012;36(2):288-305.

12. Hostetter MK. Adhesins and ligands involved in the interaction of Candida spp. with epithelial and endothelial surface. Clin Microbiol Rev. 1994;7(1):29-42.

13. Douglas LJ. Medical importance of biofilms in Candida infections. Rev Iberoam Micol. 2002;19(3):139-143.
14. Deorukhkar SC, Saini S. Why Candida species have emerged as important nosocomial pathogens? Int J Curr Microbiol App Sci. 2016;5(1):533-545.

15. Deorukhkar SC, Saini S. Medical device-associated Candida infections in a rural tertiary care teaching hospital of India. Interdisciplinary Perspectives on Infectious Diseases. 2016:5.

16. Lockhart SR. Current epidemiology of Candida infection. Clin Microbiol Newsl. 2014;36(17):131-136.

17. Seneviratne CJ, Jin L, Samaranayake LP. Biofilm lifestyle of Candida: a mini review. Oral Dis. 2008;14(7):582-590.

18. Mayer FL, Wilson D, Hube B. Candida albicans pathogenicity mechanisms. Virulence. 2013;4(2):119-128.

19. Ghannoum MA. Potential role of phospholipases in virulence and fungal pathogenesis. Clin Microbiol Rev. 2000;13(1):122-143.

20. Ying S, Chunyang L. Correlation between phospholipase of Candida albicans and resistance to fluconazole. Mycoses. 2011;55(1):50-55.

21. ElFeky DS, Gohar NM. Evaluation of Virulence Factors of Candida Species Isolated from Superficial Versus Systemic Candidiasis. Egyptian J Med Microbiol. 2016;25(1):27-36.

22. Tsang CSP, Chu FCS, Leung WK, et al. Phospholipase, proteinase and haemolytic activities of Candida albicans isolated from oral cavities of patients with type 2 diabetes mellitus. $J$ Med Microbiol. 2007;56(pt 1):1393-1398.

23. Matthews RC. Pathogenicity determinants of Candida albicans: potential targets for immunotherapy? Microbiol. 1994;140(pt 7):15051511.

24. Luo G, Samaranayake Lp, Cheung BPK, et al. Reverse transcriptase polymerase chain reaction (RT-PCR) detection of HLP gene expression in Candida glabrata and its possible role in vitro haemolysin production. APMIS. 2004;112(4-5):283-290.

25. Rodriques A, Pira Vaz C, Coste de Oliveria S, et al. Expression of plasma coagulase among pathogenic Candida species. J Clin Microbiol. 2003;41(2):2971-2974.

26. Yigit N, Aktas E, Dagistan S, et al. Investing biofilm production, coagulase and hemolytic activity in Candida species isolated from denture stomatitis patients. Eurasian J Med. 2011;43(1):27-32.

27. Gascer A, Stehr F, Kroger C, et al. Lipase 8 affects the pathogenesis of Candida albicans. Infect Immun. 2007;75(10):4710-4717.

28. Silva S, Hooper SJ, Henriques M, et al. The role of secreted aspartyl proteinases in Candida tropicalis invasion and damage of oral mucosa. Clin Microbiol Infect. 2010;17(2):264-272.

29. Soll DR. High frequency switching in Candida albicans. Clin Microbiol Rev. 1992;5(2):183-203.

30. Cannon RD, Holmes AR, Mason AB, et al. Oral Candida: Clearance, colonization or candidiasis. J Dent Res. 1995;74(5):1152-1162.

31. Mane A, Gaikwad S, Bembalkar S, et al. Increased expression of virulence attributes in oral Candida albicans isolates from human immunodeficiency virus-posiotive individuals. $J$ Med Microbiol. 2012;61(pt 2):285-290.

32. Csank C, Haynes K. Candida glabrata displays pseudohyphal growth. FEMS Microbiol Lett. 2000;89(1): 115-120. 